\title{
Efficiency of Hand Hygiene to Reduce Transient Flora on the Hands of Health Care Workers in a Tertiary Care Medical College Hospital in Coimbatore, India
}

\author{
Shreeram Astic Deshpande* and L. Arun Kumar
}

Karpagam Faculty of Medical Sciences and Research (Dr. M.G.R. Medical University) Coimbatore, Tamil Nadu-641032, India

*Corresponding author

\section{A B S T R A C T}

\section{Keywords}

Hand Hygiene, Resident flora, Steps of hygienic handwash

Article Info

Accepted:

24 February 2018

Available Online:

10 March 2018
Hand hygiene is now regarded as one of the most important element of infection control activities. Correct steps of hand hygiene as stated by WHO using hand rubs was evaluated for all study subjects. Flora of hands was studied before and after hand rub by culture. The study was conducted in a tertiary care hospital attached to Karpagam Faculty of Medical Sciences and Research in Coimbatore, between June - August, 2016. A total of 40 subjects were included out of which there were doctors, medical students, nurses, hospital and sanitary attendants, who agreed to participate in the study and were on duty in hospital premises at the time of the study.

\section{Introduction}

Most germs that cause serious infections in healthcare are spread by people's actions. Hand hygiene is a great way to prevent infections. A major concern in hospitals nowadays are Healthcare-associated Infections (HAIs) since they are responsible for increased treatment, prolonged stay and increased cost of healthcare treatment. On a large-scale aspect, these infections are also responsible for increase in the transmission of multidrug-resistant organisms in a Healthcare organization. Of every 100 hospitalized patients, at least seven in developed and 10 in developing countries will acquire health careassociated infections and in intensive care units, this figure rises to around $30 \%$.

Health care associated infections are drawing increasing attention from patients, insurers, governments and regulatory bodies. This is not only because of the magnitude of the problem in terms of the associated morbidity, mortality and cost of treatment, but also due to the growing recognition that most of these are preventable. Studies show that on average, healthcare providers clean their hands less than half of the times they should. Every patient is at risk of getting an infection while 
they are being treated for something else. Even healthcare providers are at risk of getting an infection while they are treating patients. Preventing the spread of germs is especially important in hospitals and other facilities such as dialysis centers and nursing homes.

Hand hygiene is now regarded as one of the most important element of infection control activities. In the wake of the growing burden of health care associated infections (HCAIs), the increasing severity of illness and complexity of treatment, superimposed by multi-drug resistant (MDR) pathogen infections, health care practitioners (HCPs) are reversing back to the basics of infection preventions by simple measures like hand hygiene. This is because enough scientific evidence supports the observation that if properly implemented, hand hygiene alone can significantly reduce the risk of crosstransmission of infection in healthcare facilities.

The medical community is witnessing in tandem unprecedented advancements in the understanding of pathophysiology of infectious diseases and the global spread of multi-drug resistant infections in health care set-ups. These factors, compounded by the paucity of availability of new antimicrobials have necessitated a re-look into the role of basic practices of infection prevention in modern day health care. Hence a study has been carried out to assess the efficiency of hand hygiene to reduce transient flora on the hands of health care workers in a tertiary care medical college hospital in Coimbatore and the details are provided in the following chapters.

There are two types of microbes colonizing hands: the resident flora, which consists of microorganisms residing under the superficial cells of the stratum corneum and the transient flora, which colonizes the superficial layers of the skin, and is more amenable to removal by routine hand hygiene. Transient microorganisms survive, but do not usually multiply on the skin. They are often acquired by health care workers (HCWs) during direct contact with patients or their nearby contaminated environmental surfaces (http:// www.who.int/patientsafety/en/; http://www. who.int/patientsafety/en/; Boyce and Pittet, 2002; Kampf and Kramer, 2004). The significance of hand washing in patient care was conceptualized in the early 19th century (Daniels and Rees, 1999; Labarraque, 1829; Semmelweis, 1983; Rotter, 1997). (Labarraque, 1829) provided the first evidence that hand decontamination can markedly reduce the incidence of puerperal fever and maternal mortality. Semmelweis (1983) worked in the Great hospital in Vienna in the 1840s. There were two maternity clinics in the hospital, with alternate day admission policy.

The first clinic, attended by medical students, who moved straight from autopsy rooms to the delivery suite, had an average maternal mortality rate of about 10 per cent due to puerperal fever. The second clinic, attended by midwives had a maternal mortality of only 2 per cent. The puzzled Semmelweis got a breakthrough in 1847, following the death of colleague Jokob Kolletschka, who had been accidentally got a cut by a student's scalpel while performing an autopsy. His autopsy showed a pathological condition similar to that of women drying from puerperal fever. Semmelweis concluded that some "unknown cadaverous material" caused puerperal fever. He instituted a policy of washing hands with chlorinted lime for those leaving the autopsy room, following which the rate of maternal mortality dropped ten-folds, comparable to the second clinic. Thus, he almost conducted a controlled trial, in an era when microbes were yet to be discovered and the germ theory of disease was not defined (Labarraque, 1829; Semmelweis, 1983; Rotter, 1997). 
In another landmark study in the wake of Staphylococcal epidemics in 1950s, (Mortimer et al., 1966) showed that direct contact was the main mode of transmission of $S$. aureus in nurseries. They also demonstrated that hand washing by patients' contacts reduced the level of $S$. aureus acquisition by babies.

In 1975 and 1985, the CDC published guidelines on hand washing practices in hospitals, primarily advocating hand washing with non-antimicrobial soaps; washing with antimicrobial soap was advised before and after performing invasive procedures or during care for high risk patients. Alcohol-based solutions were recommended only in situations where sinks were not available (Steere, 1975; Garner and Favero, 1985). In 1995, the Hospital Infection Control Practices Advisory Committee (HICPAC) advocated the use of antimicrobial soap or a waterless antiseptic agent for cleaning hands upon leaving the rooms of patients infected with multidrug-resistant pathogens (Hospital Infection Control Practices Advisory Committee (HICPAC), 1995). In 2002, the CDC published revised guidelines for hand hygiene (Boyce and Pittet, 2002). A major change in these guidelines was the recommendation to use alcohol based hand rubs for decontamination of hands between each patient contact (of non-soiling type) and the use of liquid soap and water for cleaning visibly contaminated or soiled hands.

A systematic review of hand washing by the Thames Valley University as part of the evaluation of processes and indicators in infection control (EPIC) study, concluded that there was a good evidence that direct patient contact resulted in hand contamination by pathogens. The EPIC study also showed the superiority of 70 per cent alcohol/ alcohol based antiseptic hand rubs (Pratt et al., 2001; Stone, 2001).
With the growing burden of HAIs, limited options of effective antimicrobials evidence supporting the role of hand hygiene in reduction of HAIs, the WHO has launched a global hand hygiene campaign. In 2005, it introduced the first Global Patient Safety Challenge "Clean Care is Safer Care (CCiSC)", as part of its world alliance for patient safety (Allegranzi et al., 2007; Magiorakos et al., 2000-09). In 2006, advanced draft guidelines on "Hand Hygiene in Health Care" were published and a suite of implementation tools were developed and tested (WHO guidelines for hand hygiene in health care (Advanced draft) Geneva: WHO, 2006).

The first Global Hand washing Day was observed on October 15, 2008. A WHO Patient Safety 2009 initiative has been established to catalyse this progress. This is the next phase of the 'First Challenge's work on CCiSC' (Allegranzi et al., 2007; Magiorakos et al., 2000-09; WHO guidelines for hand hygiene in health care (Advanced draft) Geneva: WHO, 2006; Kilpatrick et al., 2009). This initiative has seen many health care facilities registering their commitment, effectively equating to a staff of over 3.6 million people, globally. On May 5, 2009, the WHO highlighted the importance of hand hygiene and launched guidelines and tools on hand hygiene, based on the next phase of patient safety work programme "SAVE LIVES: Clean Your Hands" (http://www.who. int/patientsafety/en/; http://www.who.int/patient safety/en/).

The main aim and objectives of this study includes, Alcohol-based hand sanitizer kills most of the bad germs that make us sick and is the preferred way to clean the hands in healthcare settings in a cost effective way. Alcohol-based hand sanitizer is more effective and less drying than using soap and water, and 
does not create antibiotic-resistant superbugs. Hence the study on efficiency of hand hygiene to reduce transient flora on the hands of health care workers in a tertiary care medical hospital in Coimbatore was carried out with the following objectives

Demonstration of the correct steps of hand hygiene as stated by WHO using hand rubs to all study subjects

Assessing the hand transient flora load of health care workers such physicians, medical students, nurses and sanitary attendants before alcohol based hand sanitization

Assessing the hand transient flora load of health care workers such physicians, medical students, nurses and sanitary attendants after alcohol based hand sanitization

Comparing the effectiveness of alcohol based hand sanitization among different categories of health care workers and drawing inference for improvement

Spreading awareness on various consequences of transient skin flora and importance of proper hand hygiene based on the study results

\section{Materials and Methods}

\section{Study design}

The study was conducted in a tertiary care hospital attached to Karpagam Faculty of Medical Sciences and Research in Coimbatore between June - August, 2016.

A Total of 40 subjects were included out of which there were doctors, medical students, nurses, hospital and sanitary attendants, who agreed to participate in the study and were on duty in hospital premises at the time of the study.

\section{Educational intervention}

Training activity: Each subject taking part in the study was demonstrated the correct steps of hand hygiene- by using the conditions stated by the WHO- using hand rubs present in the hospital.

They were then requested to perform the steps and hand impressions were collected before and after hand hygiene.

\section{Teaching materials used}

WHO posters on the steps of hand hygiene were put up at various hotspots of the hospital.

\section{Evaluation toolbox}

All the subjects were administered a questionnaire for assessing their perception about hand hygiene and they were analyzed using a standard code.

\section{Sampling procedure for hand transient microflora}

The samples from the hands of all the subjects were taken by finger imprinting of all the 10 fingertips on the sheep blood agar plates (Biomerieux) on a regular day when they were on duty. A subsequent second sample was collected in the same method after hand hygiene.

The collected samples were incubated overnight and the manual colony count was done to sum up all the colonies. The upper limit for the count was100 colonies and anything above that was not counted further. Further, a comparison between the colony count before and after hand hygiene of the various subjects was done and graphical representations were created. 
Study Period: June - August 2016

Study Population: Resident doctors, medical students, nurses, hospital and sanitary attendants at a Tertiary Care Medical college Hospital, Coimbatore

Sample Size: 40 hand impressions before and after hand hygiene

Hand Rub used: Alcohol based hand rub Sterillium i.e. 2-propanol $90 \mathrm{~g} / 1000 \mathrm{ml}$ and 1propanol $60 \mathrm{~g} / 1000 \mathrm{ml}$

Instruments used: Biosafety Cabinet Level 2A, Incubator and Uniocular compound microscope.

Confidentiality: Subject confidentiality will be maintained absolutely throughout the study.

Statistical Tools: Statistical analysis will be done using SPSS20 software.

Ethical considerations: Institutional Ethical Committee clearance to be obtained and consent form attached.

\section{Observations and Results}

\section{Subject distribution}

The study comprised a total of 40subjects, which included doctors, nurses, medical students, hospital and sanitary attendant and the distribution pattern is presented in figure 1 .

\section{Hand transient flora load of health care workers before hand sanitization}

Amongst the 40 subjects about 75\% (32 subjects) were positive for transient hand flora before hand hygiene and were considered as colony +ve subjects. The rest had no colony count. The results are given in table 1 . The pie chart indicating colony positive distribution prior to hand hygiene is shown in figure 2 .
Hand transient flora load of health care workers after hand sanitization

Amongst the 40 subjects about $22.5 \%$ (9subjects) were positive for transient hand flora after hand hygiene and were considered as colony $+v e$ subjects. The rest had no colony count. The results are given in table 2 .

Comparative analysis of hand micro flora before and after sanitization among subject groups

\section{Doctors}

A total of 10 doctors were included in the study. Before appropriate hand hygiene measure, $40 \%$ (4 doctors) were positive for transient hand flora.

After appropriate measures were taken (alcohol based hand rub), none were found to be positive confirming $100 \%$ reduction in the transient hand flora due to sanitization.

\section{Medical students}

A total of 10 medical students were included in the study. Before appropriate hand hygiene measures, $100 \%$ (10 students) were found to be positive for transient hand flora.

After appropriate measures were taken (alcohol based hand rub), 9 were found to be negative for transient hand flora. Therefore there was $90 \%$ reduction in the transient hand due to sanitization. The other student had a decrease in colony count but was not completely rid of it.

\section{Nurses}

A total of 10 nurses were included in the study. Before appropriate hand hygiene measures, $80 \%$ (8 nurses) were positive for transient hand flora. 
Table.1 Hand transient flora load of health care workers before hand sanitization

\begin{tabular}{|c|c|c|c|c|c|c|c|c|c|c|c|}
\hline Subjects & \multicolumn{11}{|c|}{ Presence of microbial colonies } \\
\hline & R1 & $\mathrm{R} 2$ & R3 & $\mathrm{R} 4$ & R5 & R6 & R7 & R8 & R9 & $\mathrm{R} 10$ & $\begin{array}{l}\text { No of } \\
\text { positive } \\
\text { colonies }\end{array}$ \\
\hline Doctors & - & + & - & + & - & - & + & - & + & - & 4 \\
\hline Students & + & + & + & + & + & + & + & + & + & + & 10 \\
\hline Nurses & + & + & + & - & + & - & + & + & + & + & 8 \\
\hline $\begin{array}{l}\text { Hospital \& } \\
\text { Sanitary } \\
\text { Attendants }\end{array}$ & + & + & + & + & + & + & + & + & + & + & 10 \\
\hline
\end{tabular}

+indicates presence of microbial colonies in subjects -indicates absence of microbial colonies in subjects

Table.2 Hand transient flora load of health care workers after hand sanitization

\begin{tabular}{|c|c|c|c|c|c|c|c|c|c|c|c|}
\hline Subjects & \multicolumn{11}{|c|}{ Presence of microbial colonies } \\
\hline & $\mathrm{R} 1$ & $\mathrm{R} 2$ & R3 & $\mathrm{R} 4$ & R5 & R6 & R7 & $\mathrm{R} 8$ & R9 & R10 & $\begin{array}{l}\text { No of positive } \\
\text { colonies }\end{array}$ \\
\hline Doctors & - & - & - & - & - & - & - & - & - & - & 0 \\
\hline Students & - & - & - & + & - & - & - & - & - & - & 1 \\
\hline Nurses & - & - & - & - & - & - & + & + & + & - & 3 \\
\hline $\begin{array}{l}\text { Hospital \& Sanitary } \\
\text { Attendants }\end{array}$ & + & - & - & - & + & + & - & - & + & + & 5 \\
\hline
\end{tabular}

+indicates presence of microbial colonies in subjects

-indicates absence of microbial colonies in subjects

Figure 1. Subject distribution

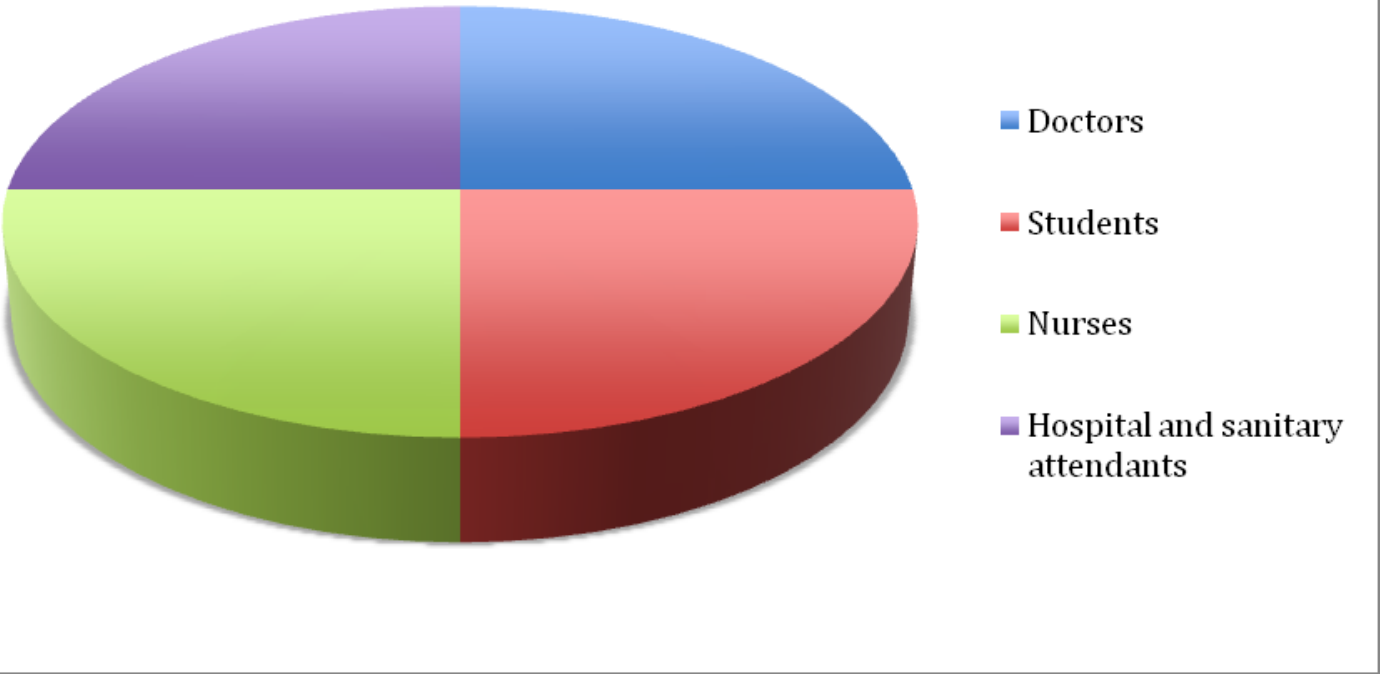


Figure 2.Colony Positive Distribution

$31 \%$

$13 \%$

\section{$31 \% \quad$ Doctors}

$25 \%$

Students

Nurses

Hospital \& Sanitary

Attendants

Fig.3 Colony +ve subjects before and after hand wash

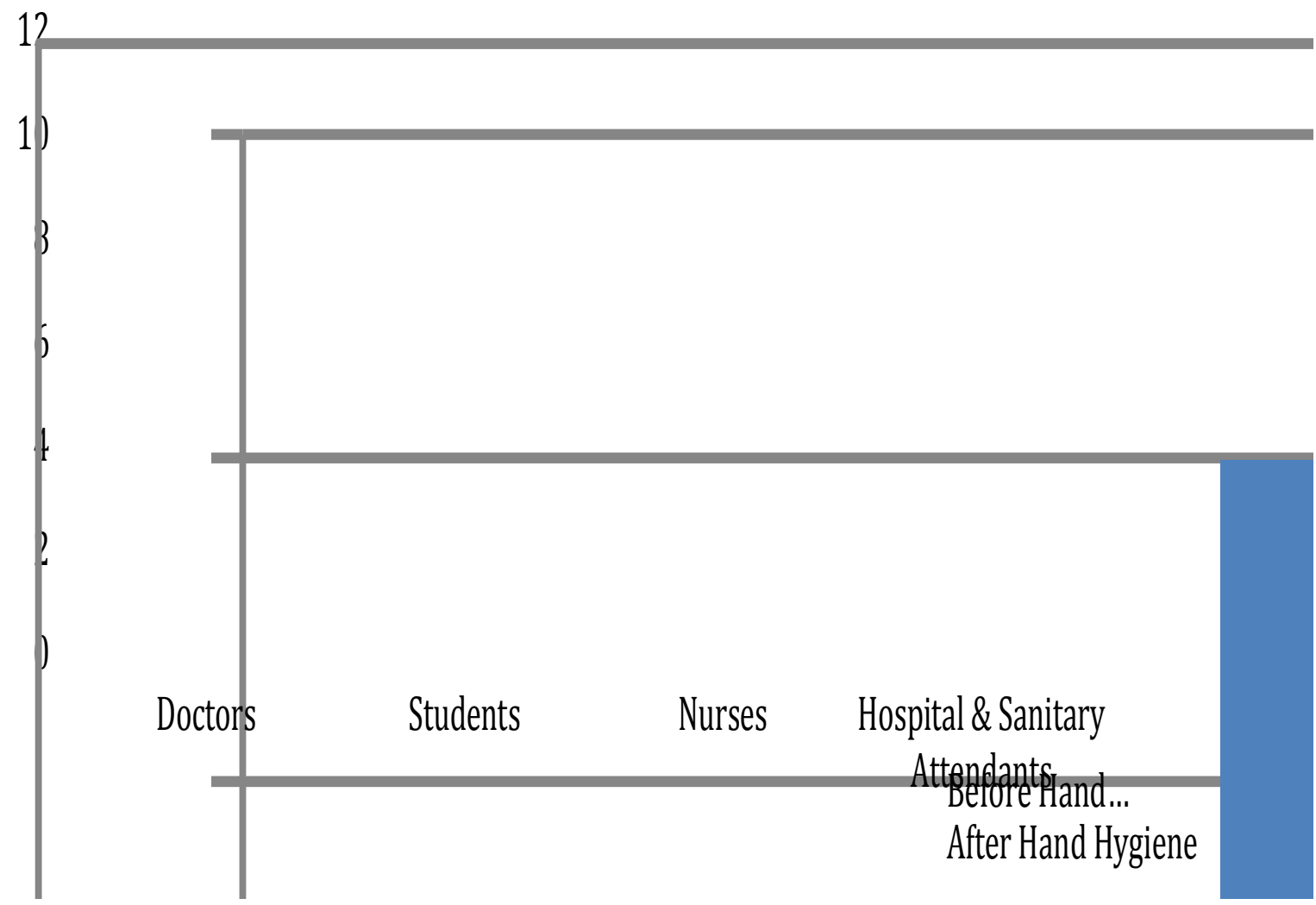




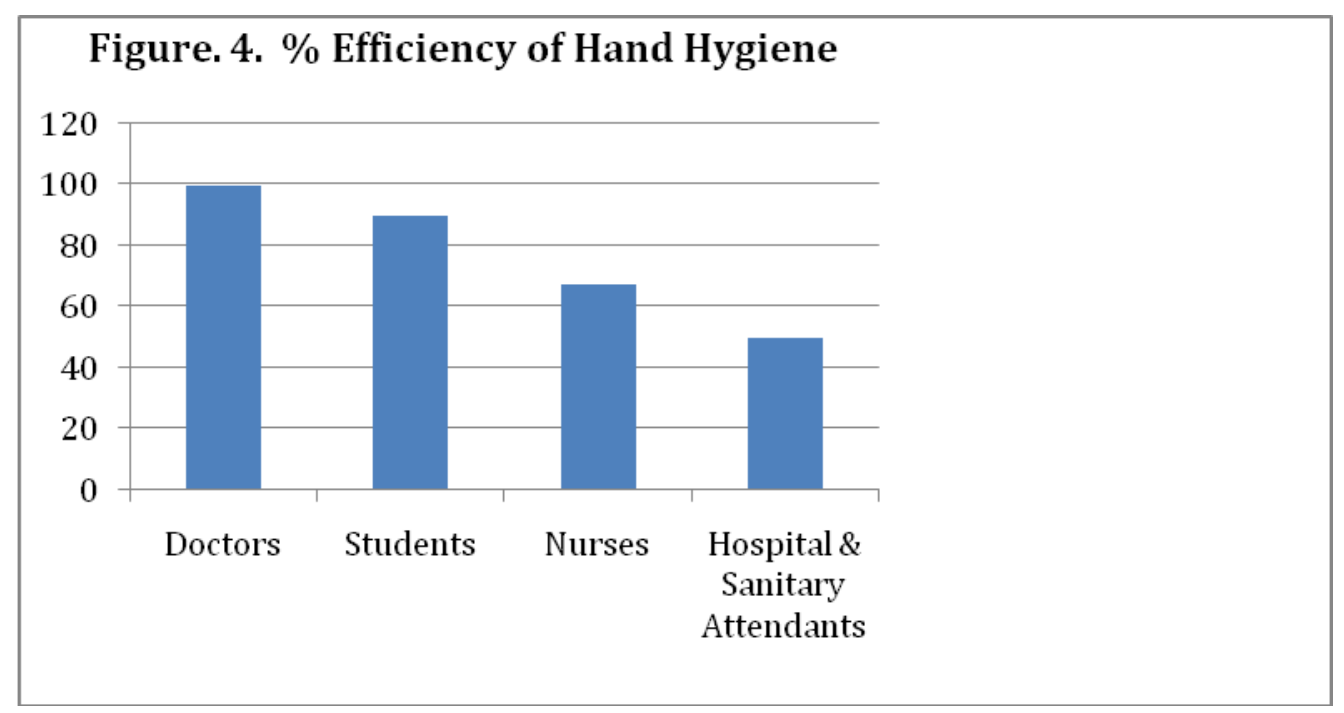

Plate.1 Blood agar medium showing the fingertip impressions of a student. The left side of the plate indicates the finger impressions before hand hygiene with high microbial colony count, while the right side of the plate is taken after hand hygiene that shows finger impressions free of transient flora

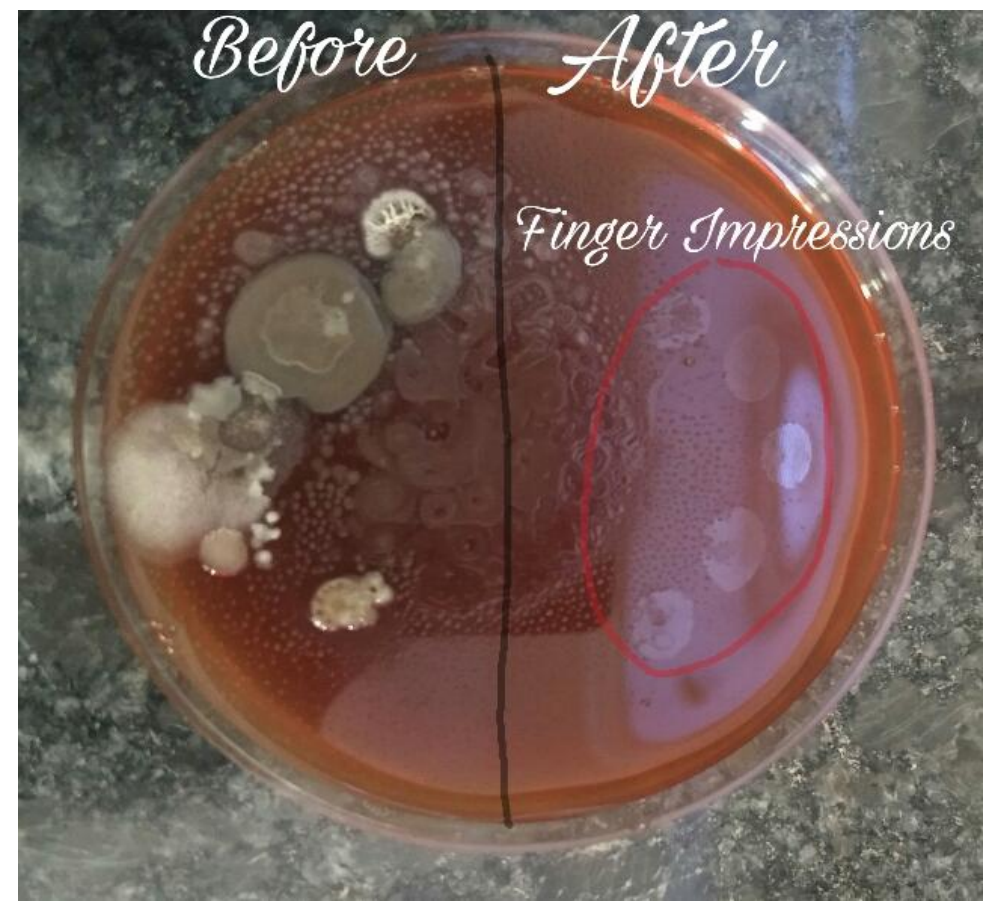

After appropriate measures were taken (alcohol based hand rub), 5 were found to be negative for transient hand flora. Therefore there was a $62.5 \%$ reduction in the transient hand flora due to sanitization. The other three had reduced colony count when compared with pre sanitization colony data.

\section{Hospital and sanitary attendants}

A total of 10 hospital staffs were included in the study. Before appropriate hand hygiene measures, $100 \%$ (10 hospital staff) were positive for transient hand flora. After appropriate measures were taken (alcohol 
based hand rub), 5 were found be to negative for transient hand flora. There was a $50 \%$ reduction in the transient hand flora positivity. The other three had reduced colony count when compared with pre sanitization colony data.

The bar graph in figure 3 indicates the amount of subjects positive for transient hand flora before and after hand hygiene.

From the bar graph (Figure 4) we can infer that the efficiency of alcohol based hand sanitization was maximum to resident doctors $(100 \%)$ followed by students $(90 \%)$ and nurses $(67.5 \%)$, while sanitary workers recorded lowest efficiency $(50 \%)$.

The difference in the efficiency of hand sanitization among study groups is mainly due to variation in performing the hand hygiene technique. Doctors who are already experienced and aware of hand hygiene performed the experiment perfectly and obtained $100 \%$ efficiency. This is in accordance with the earlier reports by Smith (Smith, 2009) who claimed that physicians generally have excellent hand hygiene skills compared to other health care workers.

Students followed the steps closely and precisely and replicated the procedure perfectly, hence obtaining 90\% efficiency. Nurses only recorded a $62.5 \%$ efficiency rate to due slight errors in performing the procedure. When discussed in detail as to the reason for this decrease in efficiency, the nurses conveyed that they had come in contact with other contaminated articles of clothing like lab coats or handkerchiefs (due to the sticky nature of the alcohol rub acting as a disturbance) before providing the second, post hand hygiene finger impressions. Some subjects, in spite of prior demonstration, followed hand hygiene procedures without removing their bangles or rings that might have contributed to less efficiency of hand sanitization.

The Hospital and sanitary attendants have the least efficiency due to two major reasons. Firstly, they are not able to comprehend the right steps of hand hygiene even after prior demonstration due to their lack of education. They are unaware of the importance of hand hygiene and do not consider it as a significant process. Secondly, most of them are not properly clothed and have untidy fingernails, which contain huge colonies of flora, which cannot completely be rid by alcohol hand rubs. The other possibility could be that the dirt load on the hands of sanitary workers might be high that requires the use of soap and water first. According to WHO guidelines on hand hygiene in health care (http://www.who.int/patientsafety/en/) skin excoriation may lead to bacteria colonizing the skin and the possible spread of blood borne viruses as well as other microorganisms. Sore hands may also lead to decreased compliance with hand hygiene protocols. This might be one another reason for the variation in the efficacy of hand sanitization among subject groups.

The presence of transient bacteria carried on the hands of HCWs is significant in persons working in the hospital as the environment and patients are commonly colonized with microorganisms. Hand hygiene has been shown to be an effective method to prevent infection by reducing the transient flora.

Our study demonstrates that the pathogens are present on the hands but could be removed effectively by proper hand hygiene using alcohol based hand rub. Somehow it was observed that despite of personal interaction, sanitary attendants were not able to follow the steps effectively that was evident by the presence of bacterial counts after the use of hand rub. 
Though there was $95 \%$ reduction in the bacterial load in majority due to hand sanitization, the ability to perform correct steps is also dependent on the level of education and attitude. Hand hygiene reflects attitudes, behaviours and beliefs. This underlines the need of repeated demonstration of the steps and importance of hand hygiene among sanitary attendants. This kind of study can be a good module for education. It provides convincing evidence that the transient flora can be reduced after appropriate hand hygiene.

The major determinants for hand hygiene product selection are antimicrobial profile, user acceptance, and cost. Hospital administrators should also take into account the acceptability of product (smell, feel, skin irritation) by the users and its allergenic potential. The available methods of hand hygiene are soap and water, alcohol based hand rubs and chlorhexidine based hand rubs. The advantage of chlorhexidine is the residual activity, but it is expensive. Soap and water are economical but the timely availability of safe running water and sterile towels are few limitations. In a busy hospital, one of the key elements in improving hand hygiene practice is the use of an alcohol based hand rub instead of washing with soap and water.

An alcohol-based hand rub requires less time, is microbiologically more effective and is less irritating to skin than traditional hand washing with soap and water. Our study has confirmed the fact that HCWs hands are grossly contaminated with pathogens and alcohol hand rubs are the easiest and most effective means of decontaminating hands and thereby reducing the rates of HAIs. Even though alcohol hand rub is effective for hand hygiene for HCWs, the success rate varies between groups and hence hand hygiene should become an educational priority to make it more effective.
One of the reasons microbes have survived in nature is due to its simple genomic framework with genetic encryptation of basic survival strategies. Hence in a hospital setting, the hands of the HCWs get colonized with transient flora comprising of pathogenic microbes. To tackle these microbes, we have to follow basic and simple protocols of infection prevention. Hand hygiene is an effective method of reducing the bacterial flora on the hands of the HCWs. The continuing medical education is one of the most important tools to reiterate the need for hand hygiene and ensure the implementation of proper hand hygiene at all categories of HCWs.

Most healthcare associated infections (HAI) are transmitted by the hands of Healthcare Workers (HCWs). It has long been known that hand hygiene among HCWs plays a central role in preventing the transmission of infectious agents. Alcohol-based hand sanitizer kills most of the bad germs that make us sick and is the preferred way to clean the hands in healthcare settings in a cost effective way. Alcohol-based hand sanitizer is more effective and less drying than using soap and water, and does not create antibioticresistant superbugs. Hence the study on efficiency of hand hygiene to reduce transient flora on the hands of health care workers in a tertiary care medical college hospital in Coimbatore was carried out between June August, 2016. The study comprised a total of 40 subjects, which included doctors, nurses, medical students, hospital and sanitary attendants. The samples from the hands of all the subjects were taken by finger imprinting of all the 10 fingertips on the sheep blood agar plates (Biomerieux) on a regular day when they were on duty. A comparison between the colony count before and after hand hygiene of the various subjects was done to assess the efficiency of hand sanitization. Amongst the 40 subjects about $75 \%$ were 
positive for transient hand flora before hand hygiene and $22.5 \%$ were positive for transient hand flora after hand hygiene. The efficiency of alcohol based hand sanitization was high to resident doctors $(100 \%)$ followed by students $(90 \%)$ and nurses $(67.5 \%)$, while sanitary workers recorded lowest efficiency (50\%). Hand hygiene reflects attitudes, behaviours and beliefs. The difference in the efficiency of hand sanitization among study groups is mainly due to variation in performing the hand hygiene technique. Doctors who are already experienced and aware of hand hygiene performed the experiment perfectly and obtained $100 \%$ efficiency. Students followed the steps closely and precisely and replicated the procedure perfectly, hence obtaining $90 \%$ efficiency. Nurses only recorded a $62.5 \%$ efficiency rate to due slight errors in performing the procedure. The Hospital and sanitary attendants have the least efficiency due to two major reasons. Firstly, they are not able to comprehend the right steps of hand hygiene even after prior demonstration due to lack of education.

They are unaware of the importance of hand hygiene and do not consider it as a significant process. Secondly, most of them are not properly clothed and have untidy fingernails, which contain huge colonies of flora, which cannot completely be rid by alcohol hand rubs. The other possibility could be that the dirt load on the hands of sanitary workers might be high that requires the use of soap and water first. Our study has confirmed the fact that HCWs hands are grossly contaminated with pathogens and alcohol hand rubs are the easiest and most effective means of decontaminating hands and thereby reducing the rates of HAIs. Even though alcohol hand rub is effective for hand hygiene for HCWs, the success rate varies between groups and hence hand hygiene should become an educational priority to make it more effective.

\section{References}

Allegranzi B, Storr J, Dziekan G, Leotsakos A, Donaldson L, Pittet D. The First Global Patient Safety Challenge "Clean Care is Safer Care": from launch to current progress and achievements. J Hosp Infect. 2007; 65(Suppl 2):115-23.

Boyce JM, and Pittet D. Guideline for Hand Hygiene in Health-Care Settings. Recommendations of the Healthcare Infection Control Practices Advisory Committee and the HICPAC/SHEA/ APIC/IDSA Hand Hygiene Task Force. Morb Mortal Wkly Rep. 2002; 51:1-44.

Daniels IR, and Rees BI. Hand washing: simple, but effective. Ann R CollSurg Engl. 1999; 81:117-8.

Garner JS, and Favero MS. CDC guideline for hand washing and hospital environmental control, 1985. Infect Control. 1986; 7:231-43.

Guide to implementation of the WHO multimodal hand hygiene improvement strategy [Accessed on August 24, 2010]. Available from: http://www.who. int/patientsafety/en/

Hospital Infection Control Practices Advisory Committee (HICPAC) Recommendations for preventing the spread of vancomycin resistance. Infect Control Hosp Epidemiol. 1995; 16:105-13.

Kampf G, and Kramer A. Epidemiologic background of Hand Hygiene and evaluation of the most important agents for scrubs and rubs. Clin. Microbiol. Rev., 2004; 17:863-93.

Kilpatrick C, Allegranzi1 B, Pittet D. The global impact of hand hygiene campaigning. Euro. Survell., 2009; 14: ii-19191.

Labarraque AG. Instructions and observations regarding the use of the chlorides of soda and lime. In: Porter J, editor. New Haven, CT: Baldwin and Treadway; 1829. 
Magiorakos AP, Suetens C, Boyd L, Costa C. National Hand Hygiene Campaigns in Europe, 2000-2009. Euro Survell. 2009; 14: ii-19191.

Mortimer EA, Wolinsky E, Gonzaga AJ, Remmelkamp $\mathrm{CH}$. Role of airborne transmission in Staphylococcal infections. BMJ. 1966; 1:319-22.

Noble WC. Dispersal of skin microorganisms. Br J Dermatol. 1975; 93:477-85.

Pratt RJ, Pellowe C, Liveday HP, Robinson $\mathrm{N}$, Smith GW, Barrett S. The EPIC project: developing national evidencebased guidelines for preventing healthcare associated infections. J Hosp Infect. 2001; 47(Suppl A):S3-82.

Rotter ML. 150 years of hand disinfectionSemmelweis' heritage. Hyg Med. 1997; 22:332-9.

Semmelweis I. Etiology, concept, and prophylaxis of childbed fever. In: Carter KC, editor. 1st ed. Madison, WI: The University of Wisconsin Press; 1983.
Smith SMS. A review of hand-washing techniques in primary care and community settings. J Clin. Nurs. 2009; 18:786-90.

Steere AC, Mallison GF. Hand washing practices for the prevention of nosocomial infections. Ann Intern Med. 1975; 83:683-90.

Stone SP. Hand hygiene: the case for evidence-based education. J R Soc Med. 2001; 94:278-81.

WHO guidelines for hand hygiene in health care (Advanced draft) Geneva: WHO; 2006 [Accessed on August 24, 2010]. World Health Organization. Available from: http://www.who.int/gpsc/tools/en/

WHO Guidelines on Hand Hygiene in Health Care. First Global Patient Safety Challenge. Clean Care is Safer Care. [accessed on August 24, 2010]. Available from: http://www.who.int/ patientsafety/en/

\section{How to cite this article:}

Shreeram Astic Deshpande and Arun Kumar, L. 2018. Efficiency of Hand Hygiene to Reduce Transient Flora on the Hands of Health Care Workers in a Tertiary Care Medical College Hospital in Coimbatore, India. Int.J.Curr.Microbiol.App.Sci. 7(03): 2630-2641. doi: https://doi.org/10.20546/ijcmas.2018.703.304 